\title{
Positron Emission Tomography/Computed Tomography before Treatment as a Predictor of ${ }^{90} \mathrm{Y}$-Ibritumomab Tiuxetan Response
}

\author{
Ryan Yudistiro ${ }^{1,2}$, Yusri Dwi Heryanto ${ }^{1}$, Sayaka Kodaira' ${ }^{1}$, Tetsuya Higuchi ${ }^{1}$, Yukiko Arisaka ${ }^{1}$, \\ Azusa Tokue ${ }^{1}$, Ayako Taketomi-Takahashi ${ }^{1}$, Norifumi Tsukamoto ${ }^{3}$, Akihiko Yokohama ${ }^{4}$, \\ Hiroshi Handa ${ }^{5}$, Hiromi Koiso ${ }^{6}$, Takuma Ishizaki ${ }^{5}$ and Yoshito Tsushima ${ }^{1}$ \\ 1 Department of Diagnostic Radiology and Nuclear Medicine, Gunma University Graduate School of Medicine, 3-39-22 Showa-machi, Mae- \\ bashi, Gunma 371-8511, Japan \\ 2 Department of Nuclear Medicine, MRCCC Siloam Hospital, School of Medicine of Universitas Pelita Harapan, Jakarta, Indonesia \\ 3 Oncology Center, Gunma University Hospital, 3-39-15 Showa-machi, Maebashi, Gunma 371-8511, Japan \\ 4 Blood Transfusion Services, Gunma University Hospital, 3-39-15 Showa-machi, Maebashi, Gunma 371-8511, Japan \\ 5 Department of Hematology, Gunma University Hospital, 3-39-15 Showa-machi, Maebashi, Gunma 371-8511, Japan \\ 6 Infection Control and Prevention Center, Gunma University Hospital, 3-39-15 Showa-machi, Maebashi, Gunma 371-8511, Japan
}

\begin{tabular}{l} 
Article Information \\
\hline Publication history: \\
Received: November 28, 2019 \\
Accepted: December 5, 2019 \\
\hline Corresponding author: \\
Ryan Yudistiro \\
Department of Diagnostic Radiology and Nuclear Medi- \\
cine, Gunma University Graduate School of Medicine, \\
3-39-22 Showa-machi, Maebashi, Gunma 371-8511, Japan \\
Tel: +81-27-220-8401 \\
E-mail: ryannuclear@gmail.com \\
\hline
\end{tabular}

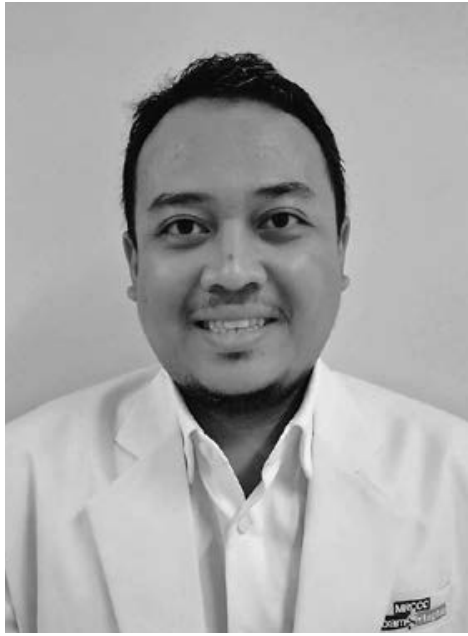

\section{Introduction}

Radioimmunotherapy (RIT) has appeared as one of the most effective treatment options for patients with relapsed or resistant indolent Non-Hodgkin's lymphoma (NHL). Although the high efficacy of RIT has been already well established, long-term toxicity, high cost, and limited availability are still potential drawbacks in the clinical setting. Therefore, a predictor of favorable outcome before RIT is needed for patient selection.

${ }^{18} \mathrm{~F}$-Fluorodeoxyglucose positron emission tomography/computed tomography (FDG-PET/CT) has been widely used and recommended as a main imaging modality in FDG-avid lymphoma. However, the role of FDG-PET/CT before treatment as a RIT response predictor has yet to be confirmed. The purpose of this study was to evaluate FDG-PET/CT before treatments as a predictor of ${ }^{90} \mathrm{Y}$-Ibritumomab tiuxetan treatment.

\section{Patients}

A retrospective study was performed in twenty patients who underwent ${ }^{90} \mathrm{Y}$-Ibritumomab tiuxetan in our institution for relapsed or resistant indolent NHL. All patients who had FDG-PET/CT at least six months before and after treatment were included. We excluded patients who had no FDG-PET/CT within six months after treatment, non-FDG-avid lymphoma, and patients who were not followed after treatment.

\section{Images analysis}

Semiquantitative parameters were obtained from a two-dimensional region of interest (2D-ROI) and three-dimensional volume of interest (3D-VOI). Adaptive-threshold of $41 \%$ was used in $3 \mathrm{D}$-VOI to measure maximum standardized uptake value (SUVmax), SUVmean, and metabolic tumor volume (MTV). Total lesion glycolysis (TLG) was obtained by multiplying SUVmean by MTV. The SUVmax and SUVpeak were obtained from 2D-ROI FDG-PET/CT images. SUVpeak was defined as an average value of SUV, which was measured in a circular ROI (fixed diameter of $1.2 \mathrm{~cm}$ ), and placed at the hottest area of the lesion. The measurable FDG-avid lesion was defined as a lesion that showed FDG uptake higher than liver and long-axis 
diameter greater than $1 \mathrm{~cm}$.

Responders were defined as patients who showed the disappearance of measurable FDG-avid lesions or decreased visual score of the residual FDG-avid lesion. Non-responders were defined as patients who showed residual FDG-avid lesions or appearance of new FDGavid lesion (s).

\section{Results}

All semiquantitative parameters except for MTV demonstrated significantly lower values in the responders compared to the non-responders $(p<0.05)$. The optimal cut-off values identified by ROC analysis for SUVmax using 3D-VOI and 2D-ROI were 5.35 and 5.15, respectively, with these values having sensitivity and specificity of $83.3 \%$ and $71.3 \%$. Meanwhile, the optimal cut-off values for SUVpeak and TLG was 4.35 and 19.5 grams, respectively, with the sensitivity and specificity for SUVpeak being $83.3 \%$ and $92.9 \%$ and both being 100\% for TLG. (Fig. 1)

\section{ROC curve of FDG-PET/CT before RIT}

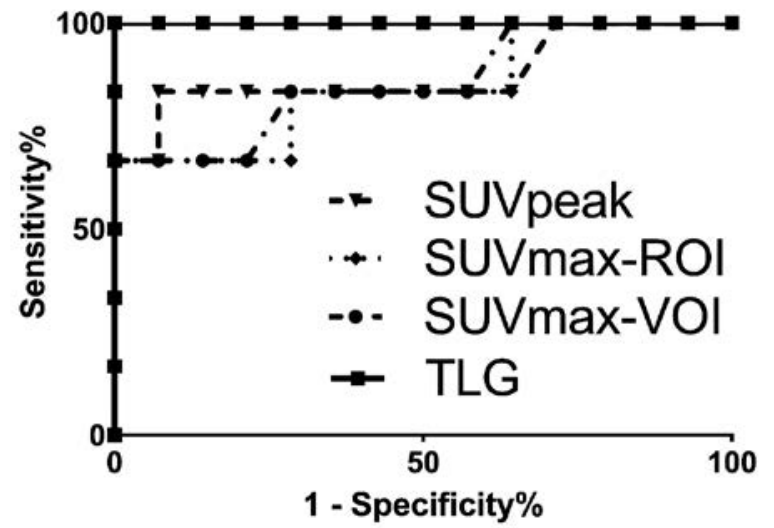

Fig. 1 ROC curve of FDG-PET/CT before RIT

\section{Discussion}

It has been reported that higher FDG-avidity was correlated with more aggressive lesions, and that this may be due to overexpression of glucose transporter-3. We suspected that our findings might reflect a transformation of lymphoma in non-responder patients to more aggressive subtypes, although we did not have direct histopathological confirmation. Our average SUVmax was slightly lower than other studies that showed SUV of $>$ 10 as a threshold in differentiating between indolent and aggressive NHL. Lymphoma cells in mass lesions may not be homogeneous, possibly containing more aggressive cells generated by transformation. The presence of these aggressive cells is likely to determine the potential tumor aggressiveness, rather than the total number of lymphoma cells or the total volume of tumors. In the current study, SUVs and TLG also reflect lesion viable cell density that is useful in predicting treatment response; meanwhile, MTV was not useful in predicting treatment response. This difference may partially support our suggestion concerning transformation, and our results were consistent with previous reports. From our study, we suspected that patients with SUVmax, SUVpeak, and TLG of FDG-PET/CT above the cut-off values before treatment may not be appropriate candidates for RIT, and other treatment options should be considered.

\section{Conclusion}

We found that the semiquantitative parameters of FDG-PET/CT before RIT treatment had significantly lower values in the responders compared to the non-responders, confirming that FDG-PET/CT before treatment may be a valuable imaging biomarker as an early predictor of RIT response. 